総 説

\title{
Theoretical approaches on the growth strategy of plants in unpredictable environments
}

\author{
Satoki SAKAI \\ Biological Institute, Graduate School of Science, Tohoku University, Aoba, Sendai 980-77, \\ JAPAN
}

\begin{abstract}
I showed two models for growth strategies of plants growing in unpredictable environments. In the first model, I analyzed the evolutionarily stable sapling growth waiting for future gap formation under closed canopy. In the second model, I analyzed the optimal resource allocation strategies to ramets (daughter plants), rhizomes (or other horizontal stems, such as stolons) and seeds for plants growing in a spatially varying environment.
\end{abstract}

Key words: sapling growth, gap formation, foraging by clonal plants, resource allocation model

\section{Introduction}

Plants often grow in environments where parameters that affect their fitnesses are unpredictable. For example, saplings of canopy trees under closed forest cannot grow into adult trees without improvement of light condition by gap formation, but gap formation is not predictable for those saplings. For clonal plants that forage favorable patches by rhizome or stolon elongation, whether or not a rhizome (stolon) encounters a favorable patch is also unpredictable. What is then the optimal growth strategies in these unpredictable environments? Should a sapling grow vertically for height advantage to gap occupancy after gap formation in anticipation of near future gap formation, or should it develop an effective photosynthetic system at the present height to survive for a long period under closed canopy? Should a clonal plant elongate many short rhizomes or a few long rhizomes to search favorable patches? In the present paper, I illustrate the analyses on the optimal growth strategies of plants in unpredictable environments by two examples of mathematical models (Sakai 1995a, b).

\section{Growth strategy of saplings under closed canopy}

Sakai (1995) developed a model for the growth strategy of a sapling waiting for a gap formation under closed canopy. I assumed that a gap is formed randomly at a rate of $p$ per year, and a sapling can grow into an adult tree if it successfully occupies a gap. However, a sapling dies at a rate of $m$ per year under closed canopy. A sapling consists of a trunk and a 
photosynthetic part. The photosynthetic part includes leaves and branches which reduce self-shading. Annual photosynthates produced by the photosynthetic part are allocated to these two parts. A fraction of the photosynthetic part is lost annually because of leaf shedding and constant branch loss accompanying with vertical growth. The sapling can produce more resources under the closed canopy if it has a larger photosynthetic part, but once a gap is formed, the sapling can occupy the gap more successfully if it has a larger trunk.

Let $S(t)$ and $F(t)$ be the sizes of the trunk and the photosynthetic part at a time $t$, respectively. Let $V[F(t)]$ be the photosynthates produced by the photosynthetic part at $t$. The growth of the trunk and the photosynthetic part is described as

$$
\begin{aligned}
& \frac{d S}{d t}=u(t) V[F(t)], \\
& \frac{d F}{d t}=[1-u(t)] V[F(t)]-c F(t),
\end{aligned}
$$

where $u(t)(0 \leq u \leq 1)$ is the fraction of photosynthates allocated to the trunk at $t$, and $c(0<c \leq 1)$ is a positive constant, which represents the annual loss rate of the photosynthetic part.

The probability that a sapling either experiences a gap formation or it dies in a year is $m+p$ if the probability that both occurs at the same time can be neglected. Hence, the probability that a sapling survives until $t$ without gap formation is $\exp \{-(m+p) t\}$, and the probability that this sapling meets a gap formation at $t$ is $p \exp \{-(m+p) t\}$. The fitness $(\phi)$ of the sapling is thus defined as

$$
\phi=\int_{0}^{\infty} p \exp [-(m+p) t] J[S(t)-\bar{S}] d t,
$$

where $J[S(t)-\bar{S}]$ is the reproductive success of a sapling with size $S(t)$ when a gap is formed at $t$, and $\bar{S}$ is the mean trunk size of the competitors within the same gap. $J$ increases with the difference of the trunk size of the sapling, $S$, from the mean trunk size of the competitors, $\bar{S}$.

Figure 1 shows an example of the evolutionarily stable sapling growth which consists of three phases. The first or the first two phases may be absent depending on the parameters. In the first phase $\left(0<t<t_{1}\right)$, the sapling allocates all annual photosynthates to its photosynthetic part, and the trunk does not grow during this phase. In the second phase $\left(t_{1}<t<t_{2}\right)$, the sapling allocates annual photosynthates both to the trunk and to the photosynthetic part, and the sizes of both parts increase with $t$. In the third phase $\left(t_{2}<t<\infty\right)$, the sapling also allocates annual photosynthates both to 
the trunk and to the photosynthetic part. However, the resource allocation ratio and the size of the photosynthetic part do not change because the sapling allocates photosynthates to this part only to make up for the loss (i.e., $u^{*}=1-c F^{*} / V^{*}$, so that $d F^{*} / d t=0$ ), and only the trunk size increases during this phase.

In the second and the third phases of the growth schedule, saplings should allocate photosynthates more to their photosynthetic parts to have larger sizes of these parts if larger trunks are more strongly advantageous in competition in gaps. This is because a sapling with large $u$ and small $F$ cannot have a larger trunk than a sapling with small $u$ and large $F$ except for a brief period after germination (Fig. 2). Namely, sapling with large $u$ can have a larger trunk than a sapling with small $u$ during a brief period after germination. However, the former sapling cannot have a larger photosynthetic part due to its small $u$. The latter sapling can produce larger photosynthates because of its larger photosynthetic part, and as a result, this sapling can allocate more photosynthates to its trunk even if its $u$ is small. Thus, saplings should have small $u$ and large $F$ in terms of future trunk growth. On the other hand, saplings should allocate photosynthates more to their trunks if mortality is larger or if probability of gap formation is larger. This is because the probability that a sapling survives until $t$ without gap formation $[\exp \{(-m+p) t\}]$ decreases with increasing $m$ and $p$, and hence the advantage of future trunk growth is small if $m$ or $p$ is large. Hence, saplings emphasize trunk growth in the short period after germination with large $u$ at the expense of large trunk growth in future years. Thus, the growth schedule changes with the changes in the relative advantages of 1) having a large trunk in the short period after germination, and 2) having a large trunk in future years.

\section{Growth strategy of clonal plants}

Sakai (1995b) developed an optimal-resource allocation model to ramets, rhizomes, and seeds for plants growing in a spatially varying environment. I assumed that favorable patches are distributed randomly in the habitat, and a plant disperses its offspring through ramet and/or seed production. In ramet production, the parent might search for patches by elongating rhizomes (or stolons) which grow in various directions. If a rhizome encounters a patch, the rhizome forms a ramet in that patch, whereas if not, the rhizome must endure placing the ramet outside patches. In addition, it is also possible that the parent produces daughter ramets next to the parent without patch searching (i.e., produces ramets without rhizomes). In seed production, seeds are dispersed randomly over a wide area, and whether a seed is dispersed to a patch depends on the relative area of patches to the total area of the habitat.

The parent allocates $T$ resources to reproduction:

$$
\begin{gathered}
T=\left(c_{r}+R\right) N+c_{s} S, \\
-23-
\end{gathered}
$$


where $R, N$, and $S$ are the mean resource allocation per rhizome, the mean number of daughter ramets, and the mean number of seeds, respectively, and positive constants $c_{r}$ and $c_{s}$ are the respective costs of producing one ramet and one seed.

The fitness of the parent is defined as

$$
\phi=\frac{e N}{1+b N} g(R)+h(S),
$$

where $e$ represents the relative potential probability of ramet to seed establishment (the larger $e$ is, the larger potential probability of ramet establishment relative to seed establishment). The first term in the righthand side represents the fitness through ramet production, where $g(R)$ represents the expected success per ramet when $R$ resources are allocated per rhizome. $g(R)$ increases with $R$ because the probability that a rhizome encounters a favorable patch increases with $R$. However, as expressed by $N /(1+b N)$, the number of daughter ramets established becomes saturated as the ramet number becomes large because of sib-competition between daughter ramets. The second term in the right-hand side represents the number of daughter plants established through seed production. $h(S)$ depends on the number of favorable patches per unit area, $\rho$, because the probability that a seed falls in a favorable patch increases with $\rho$.

Figure 3 shows the parameter regions of different optimal resource allocation patterns depending on $f_{2} / f_{1}$, the relative potential probability of offspring establishment in patches to outside patches, and $\rho$, the number of patches per unit area. The following arguments may be helpful to understand these results. Namely, parents can place offspring in patches with a higher probability by rhizome elongation than by seed dispersal, because rhizomes can search patches whereas seeds are dispersed randomly without patch selection. In addition, the potential probability of ramet establishment is much larger than that of seed establishment. However, the cost per rhizome (and its ramet) is much larger than the cost per seed. Thus, I can summarize the following general trends. 1) Parents should emphasize rhizome elongation if patches are more favorable $\left(f_{2} / f_{1}\right.$ is larger). This is because the increasing favorability of patches favors rhizome elongation, by which parents can place ramets in patches selectively, more strongly than seed production. 2) Parents should also emphasize rhizome elongation if there are more patches per unit area ( $\rho$ is larger) because parents need not elongate longer rhizomes if $\rho$ is larger. 3) Parent should emphasize seed production if the relative potential probability of ramet to seed establishment is smaller ( $e$ is larger).

Then in Figs. 3b and 3c, both rhizome elongation and seed dispersal can be advantageous because of the moderate values of $e$ in these figures. Parents elongate rhizomes to place their ramets in favorable patches 
selectively, with smaller resource allocation per rhizome, if $f_{2} / f_{1}$ and $\rho$ are larger. In addition, because $e$ is larger in Fig. 3b than in Fig. 3c, parents emphasize rhizome elongation rather than seed production in Fig. $3 \mathrm{~b}$, whereas they emphasize seed production rather than rhizome elongation in Fig. 3c. On the other hand, parents do not produce seeds if $e$ is very large (Fig. 3a), whereas they produce seeds without rhizome or ramet production if $e$ is very small (Fig. 3d). However, as shown in Figs. 3b and 3d, parents decrease seed production and produce ramets without rhizomes if $f_{2} / f_{1}$ is very smaller and $\rho$ is large. Thus, the optimal growth strategies of plants differ largely depending on parameter values of $e, f_{2} / f_{1}$, and $\rho$.

\section{References}

Sakai, S. 1995a. Evolutionarily stable growth of a sapling which waits for future gap formation under closed canopy. Evol. Ecol. 9: 444-452.

Sakai, S. 1995b. Optimal resource allocation to vegetative and sexual reproduction of a plant growing in a spatially varying environment. J. theor. Biol. 175: 271-282. 


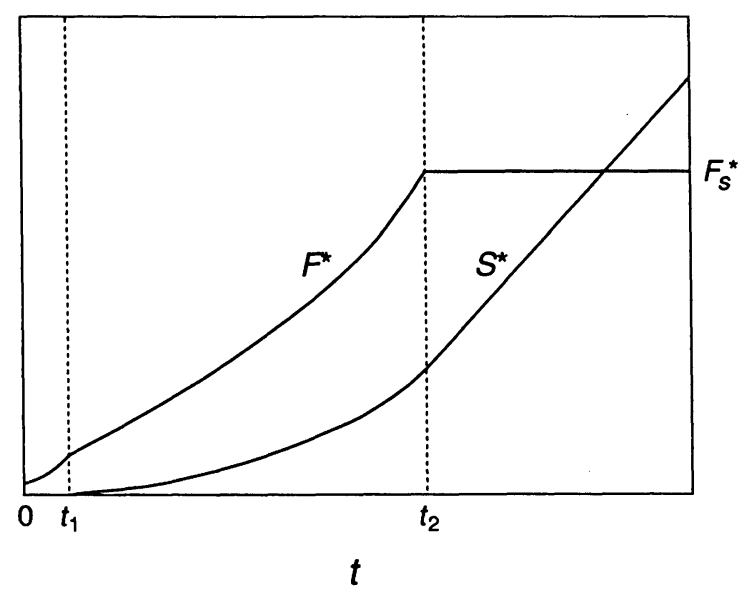

Fig. 1. An example of sapling growth. $S^{*}$, size of the trunk; $F^{*}$, size of the photosynthetic part; $F_{s}{ }^{*}$, size of the photosynthetic part in the third phase; $t_{1}$, beginning of the second phase; $t_{2}$, beginning of the third phase. (Reproduced from Sakai, 1995a).

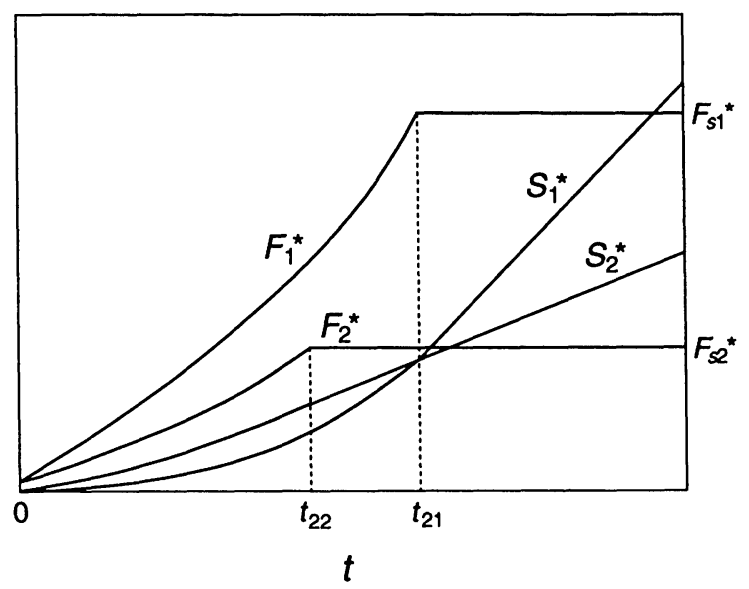

Fig. 2. Changes in the sapling growth depending on the degree in the advantage of a large trunk in competition in gaps, $d$, annual mortality, $m$, and the annual probability of gap formation, $p$. Symbols with the suffix $1\left(S_{1}{ }^{*}, F_{1}{ }^{*}, F_{s 1}{ }^{*}, t_{21}\right)$ show the growth of a sapling with a larger $d$, a smaller $m$, or a smaller $p$, whereas symbols with the suffix $2\left(S_{2}{ }^{*}, F_{2}{ }^{*}, F_{s 2}{ }^{*}, t_{22}\right)$ show the growth of a sapling with a smaller $d$, a larger $m$, or a larger $p . S^{*}$, size of the trunk; $F^{*}$, size of the photosynthetic part; $F_{s}{ }^{*}$, size of the photosynthetic part in the third phase; $t_{2}$, beginning of the third phase. The first phase is assumed to be absent for simplicity. (Reproduced from Sạai, 1995a).

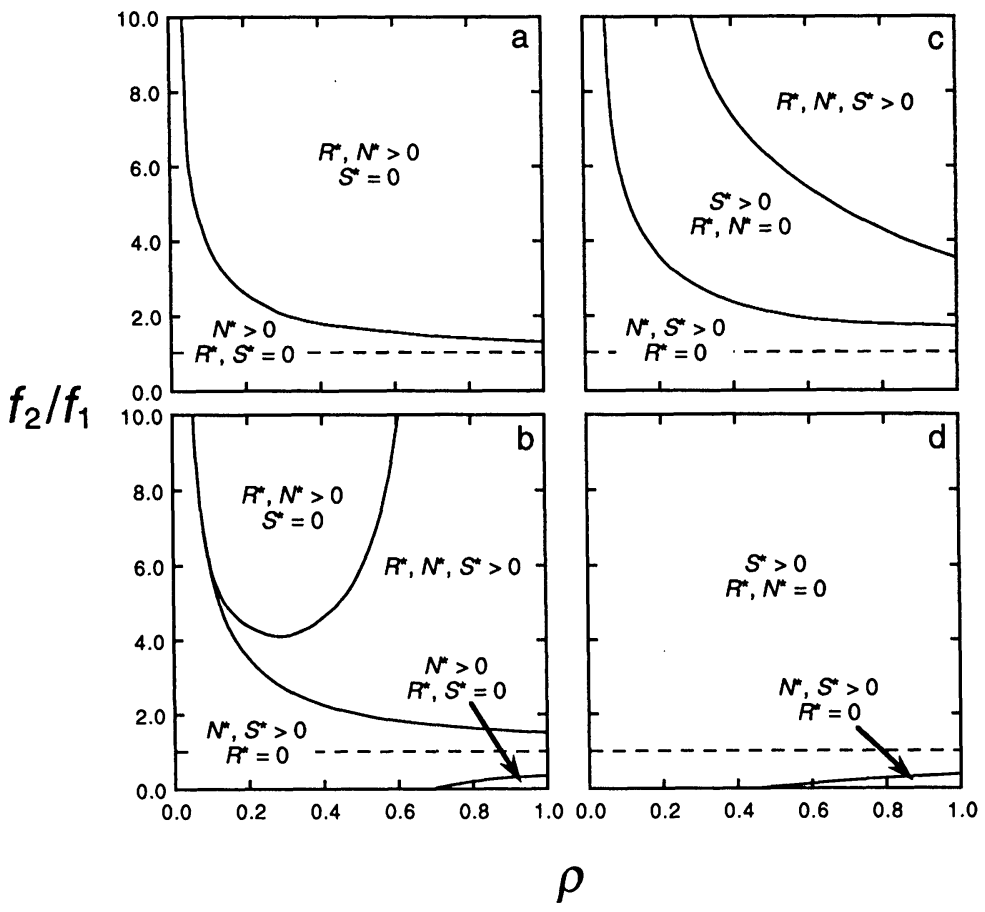

Fig. 3. The parameter regions of different optimal resource allocation patterns depending on $f_{2} / f_{1}$, the relative potential probability of offspring establishment in patches to outside patches, and $\rho$, the number of patches per unit area. The dotted lines indicate where $f_{2} / f_{1}=1$. $e$, the relative potential probability of ramet to seed to establishment, is 333.3, 83.3, 29.4, and 12.5 in a), b), c) and d), respectively. (Reproduced from Sakai, 1995b). 